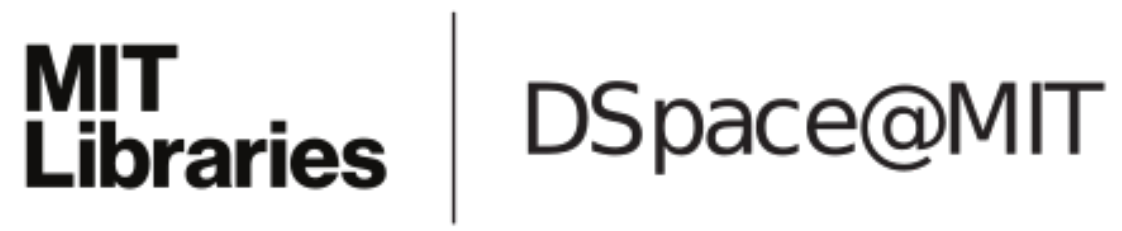

\author{
MIT Open Access Articles
}

Effects of firing angle imbalance on 12pulse rectifiers with interphase transformers

The MIT Faculty has made this article openly available. Please share how this access benefits you. Your story matters.

Citation: Perreault, D.J., and J.G. Kassakian. "Effects of Firing Angle Imbalance on 12-Pulse Rectifiers with Interphase Transformers." IEEE Trans. Power Electron. 10, no. 3 (May 1995): 257262. (C) 1995 IEEE

As Published: http://dx.doi.org/10.1109/63.387989

Publisher: Institute of Electrical and Electronics Engineers (IEEE)

Persistent URL: http://hdl.handle.net/1721.1/86988

Version: Final published version: final published article, as it appeared in a journal, conference proceedings, or other formally published context

Terms of Use: Article is made available in accordance with the publisher's policy and may be subject to US copyright law. Please refer to the publisher's site for terms of use. 


\title{
Effects of Firing Angle Imbalance on 12-Pulse Rectifiers with Interphase Transformers
}

\author{
David J. Perreault, Student Member, IEEE, and John G. Kassakian, Fellow, IEEE
}

\begin{abstract}
Firing angle or source imbalances between 6-pulse bridges comprising a 12-pulse rectifier lead to current imbalance, due to the finite magnetixing inductance of the interphase transformer. The magnitude of the imbalance is limited by the negative feedback produced by load regulation of the 6-pulse groups. This paper uses both an averaged model and a piecewiselinear simulation to obtain a quantitative understanding of this effect. The averaged model is used to predict steady-state current shifts and transient behavior. The piecewise-linear model of the system is then used to verify and extend the results via computer simulation. It is shown that the current imbalance can be accurately predicted by the averaged model, making the model suitable for both feedback control and interphase transformer design.
\end{abstract}

\section{INTRODUCTION}

$\mathbf{F}$ IGURE 1 shows a 12-pulse rectifier composed of two 6pulse bridges paralleled through an interphase transformer (IPT). The two three-phase source sets that supply the 6pulse bridges are phase displaced by $30^{\circ}$ with respect to each other, while the IPT is designed to force equal dc currents in each bridge by absorbing the instantaneous difference between the output voltages of the bridges. As long as there is no average difference between the bridge voltages $v_{d 1}$ and $v_{d 2}$, the IPT will function properly. In the presence of an average voltage, however, the IPT will saturate, causing each bridge to operate in a discontinuous conduction mode in which each rectifier conducts for only $60^{\circ}$ and carries the full load current. This problem is especially acute in phasecontrolled converters, where an average difference can occur when the firing angles of the two bridges are unequal. This paper analyzes the current imbalance problem, demonstrates that the negative feedback implicit in a converter with load regulation, i.e., commutating impedance, provides corrective action, and quantifies the extent of this correction.

Unbalanced operation of the 6-pulse bridges in a 12-pulse converter has been studied by others. Bennell described the dependence of current balance on mismatch and coupling between line transformer secondaries [1], while Evans has analyzed the interaction between supply harmonics and converter load balance [2]. Subbarao et al. considered the problem of firing angle imbalance in 12-pulse rectifiers configured as series-connected 6-pulse bridges, but inherent to this configuration is the independence of bridges and absence of an interphase transformer [3]. Others have investigated the effects

Manuscript received November 24, 1993; revised January 13, 1995.

The authors are with the Massachusetts Institute of Technology Laboratory for Electromagnetic and Electronic Systems, Cambridge, MA 02139 USA.

IEEE Log Number 9410516.

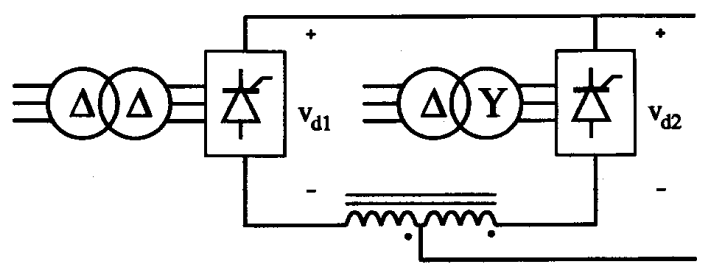

Fig. 1. A twelve-pulse rectifier with interphase transformer.

of firing angle imbalance on line transformer operation [4], [5]. However, these analyses do not consider the presence of commutating reactance and thus are not applicable to the present case. The only work to have addressed the problem of firing angle imbalance in phase-controlled converters with interphase transformers is that of Tanaka et al. [6], who present a piecewise model for simulating the current balance between converters, and use it to analyze some examples. However, the focus of this excellent paper is the development of a feedback control system for balancing bridge currents, and not the analysis of the general case.

In this paper, we derive a new averaged model for the 12pulse rectifier system which directly predicts the effects of unequal firing angles. The effects of unbalanced turns ratios and commutating reactances are also analyzed. We then use a piecewise linear simulation of the system to verify the results and extend them to cases where averaging is not justified. Both the analytic and simulation-based results are presented in a normalized form. The value of these normalized results is that they simplify the IPT design process by allowing the designer to easily predict the conditions under which the IPT must operate. Furthermore, the averaged model is a useful tool for feedback control design, since it models the averaged dynamics of the system.

\section{MODELS}

The circuit being studied is shown in Fig. 2. The inductor $L_{\mu}$ represents the magnetizing branch of the IPT model. The source inductors $L_{c}$ represent transformer, filter, and line impedances. As in [6], we assume a constant current load, ideal switches, and negligible winding resistance in the transformers. We first derive an averaged model that yields approximate analytic expressions for the imbalance between the currents of the two 6-pulse bridges. These analytic expressions provide valuable insight into the parametric dependence of the unbalance. Then, using a piecewise-linear model, we simulate the circuit of Fig. 2 to validate the results obtained 


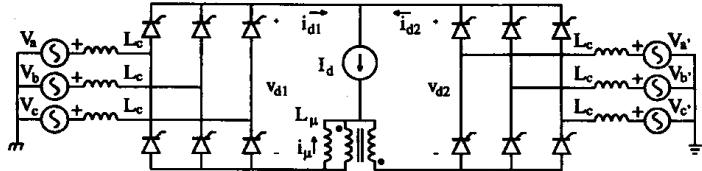

Fig. 2. A circuit model for the 12-pulse rectifier.

using the averaged model. The simpler averaged model can then be used with confidence to study a variety of specific operating conditions.

\section{A. The Averaged Model}

We find an expression for the difference between the average output currents of the two six-pulse bridges by considering an approximation to the system in which each bridge carries a local average current and delivers a local average output voltage. The local average is defined as the moving average over a finite period which is short compared to the system dynamics. This leads to a solution which is exact in the limiting case of an infinite magnetizing inductance.

Consider the behavior of the circuit of Fig. 2 under a slight differential in the firing angles of the two 6-pulse bridges. We will assume that bridge 2 firing signals lag bridge 1 signals by $\Delta \alpha$, with $\alpha_{1}$ denoting the firing angle of bridge 1. The firing angle differential generates a dc component in the voltage across the IPT. This causes the magnitude of the magnetizing current $i_{\mu}$ to increase with time, resulting in unequal bridge currents-the higher voltage bridge carrying the greater load current. As the imbalance increases, the load regulation produced by $L_{c}$ will cause the average output voltage of the higher voltage bridge to decrease, and that of the lower voltage bridge to increase. At some point (presuming the IPT does not saturate), the voltage difference caused by the firing angle differential will be completely compensated by the effect of load regulation. There will then be no dc voltage across the IPT, and a steady-state current imbalance will have been established.

We now examine the averaged behavior more formally. We will work under the assumption that the magnetizing current ripple is small (i.e., that the magnetizing inductance, $L_{\mu}$, is large), allowing the magnetizing current, $i_{\mu}$, to be replaced by its local average value, $\bar{i}_{\mu}$. The consequences of relaxing this constraint on the ripple in $i_{\mu}$ will be considered in a later section. For our purposes, the local average of a variable will be defined as its average over the bridge ripple period:

$$
\bar{x}(t)=\frac{3 \omega}{\pi} \int_{t-(\pi / 3 \omega)}^{t} x(\tau) d \tau .
$$

(In the periodic steady state, the local average, $\bar{i}_{\mu}(t)$, equals the average, $I_{\mu}$.) Under the additional constraint that $\Delta \alpha$ varies more slowly than one half the ripple frequency, we can use circuit averaging to predict the local-average dynamics of the current balance [7]. In an averaged sense, a bridge with load regulation has the same terminal characteristics as an equivalent voltage source with series resistance [8]. Representing the bridges this way leads to the averaged model of Fig. 3, in which $V_{s}$ is the peak line-to-line source voltage.

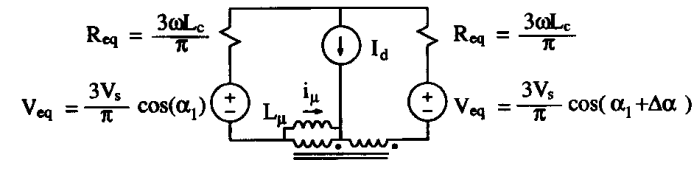

Fig. 3. An averaged model for the 12-pulse rectifier.
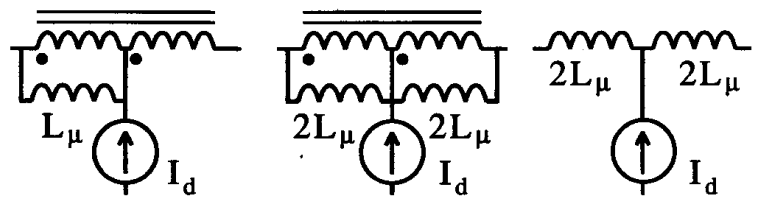

Fig. 4. Equivalent IPT models valid for constant output current, $I_{d}$.

The assumption of small magnetizing current ripple used above is forced by the fact that the load regulation characteristic employed in the model assumes a constant output current for each bridge. The slow variation restriction on $\Delta \alpha$ in the model stems from the fact that the real system can respond no faster than the ripple frequency rate to changes in $\Delta \alpha$. Also, note that the averaged model is only valid as long as both bridges are operating in mode I (nonoverlapping commutations within a bridge). While transition away from this condition is difficult to predict when ripple is considered, a rough estimation can be made by calculating the commutation angle of each bridge under the assumption of constant output current. For a 6-pulse bridge, this calculation yields a commutation angle, $\mu$, of

$$
\mu=\cos ^{-1}\left(\cos (\alpha)-\frac{2 X_{c} I_{d c}}{V_{s}}\right)-\alpha
$$

As long as the magnetizing inductance is sufficiently large to limit the interactions between converters, the model remains valid until the commutation angle of one of the bridges exceeds $\pi / 3$.

An analysis of the circuit model of Fig. 3 shows that the system will show a first-order response to a slowly varying $\Delta \alpha$ with an $L / R$ time constant of

$$
\tau=\frac{2 \pi L_{\mu}}{3 \omega L_{c}}
$$

The differential-mode response of the circuit is seen more easily when the substitutions of Fig. 4 are used to make the circuit symmetric. Solving for the average magnetizing current resulting from a constant $\Delta \alpha$ and normalizing the equation to the total output current $I_{d}$ yields

$$
\frac{\bar{i}_{\mu}}{I_{d}}=\frac{-2}{\left(\frac{X_{c} I_{d}}{V_{s}}\right)} \sin \left(\alpha+\frac{\Delta \alpha}{2}\right) \sin \left(\frac{\Delta \alpha}{2}\right) .
$$

Here we have expressed the relative current imbalance in terms of the firing angle $\alpha_{1}$, the firing angle differential $\Delta \alpha$, and the reactance factor $X_{c} I_{d} / V_{s}$. The implication of this equation is that (ignoring ripple and saturation) when $\bar{i}_{\mu} / I_{d}=1$, the entire load current is carried by one bridge since the local average bridge currents are $\bar{i}_{d 1}=\frac{1}{2}\left(I_{d}-\bar{i}_{\mu}\right)$ and $\bar{i}_{d 2}=\frac{1}{2}\left(I_{d}+\bar{i}_{\mu}\right)$. Thus, we have an analytic expression, (4), 
that approximates the average load current imbalance expected in a system with a constant firing angle imbalance.

Using the averaged model of Fig. 3 to analyze the effects on output voltage of the unbalanced currents predicted by (4) yields

$$
\left\langle v_{d}\right\rangle=\frac{3 V_{s}}{\pi}\left[\frac{\cos \left(\alpha_{1}+\Delta \alpha\right)+\cos \left(\alpha_{1}\right)}{2}-\frac{X_{c} I_{d}}{2 V_{s}}\right] .
$$

This equation is similar to the expression for $\left\langle V_{d}\right\rangle$ under balanced conditions, i.e.,

$$
\left\langle v_{d}\right\rangle=\frac{3 V_{s}}{\pi}\left[\cos (\alpha)-\frac{X_{c} I_{d}}{2 V_{s}}\right] .
$$

The only difference is that $\cos (\alpha)$ in (6) is replaced by the average of $\cos \left(\alpha_{1}\right)$ and $\cos \left(\alpha_{1}+\Delta \alpha\right)$ in (5).

Simple variations on the model of Fig. 3 can be used to predict the effects of various other conditions on current balance. For example, because the desired turns ratios of the transformers cannot be met exactly, there is often a voltage imbalance between the transformer secondaries of the two bridges [1]. Using unbalanced input voltages in the model, we find an average magnetizing current results for balanced firing angles $(\Delta \alpha=0)$ :

$$
\frac{\bar{i}_{\mu}}{I_{d}}=\frac{(k-1) \cos (\alpha)}{\left(\frac{X_{c} I_{d}}{V_{s}}\right)}
$$

where $k$ is the ratio of the bridge 2 to bridge 1 ac source voltages, i.e., $k=V_{s 2} / V_{s 1}$.

Similarly, if the firing angles and turns ratios are correct, but the commutating inductances of the two bridges are not equal, the resulting dc magnetizing current is

$$
\frac{\bar{i}_{\mu}}{I_{d}}=\frac{L_{c 1}-L_{c 2}}{L_{c 1}+L_{c 2}} .
$$

This result agrees with the observations of Bennell [1].

Note that the bridge currents that result from these equations are also correct if individual output inductors are used for current sharing, as long as they are of sufficient size to make the assumption of small current ripple valid. The transformed circuit of Fig. 4 exhibits the same incremental behavior as the original, as long as the load current is fixed. Thus, the two cases are equivalent if the output current $I_{d}$ is constant, and $L_{s}=2 L_{\mu}$.

\section{B. The Piecewise Model}

While the averaged analysis yields a convenient analytic expression for the individual 6-pulse bridge currents, it assumes that all variables are accurately represented by their local averages. To capture circuit behavior more precisely, and to determine the limits of the averaged model, we turn to time domain simulation of the system using piecewise linear models.

Treating the thyristor switches as ideal, we can analyze the circuit behavior in terms of the circuit response during individual switch configurations, with each switch configuration being a set of switch states (on or off). Associated with each switch configuration is a set of state equations and a set of validity conditions. The state equations describe the trajectory of the system during a single switch configuration, while the validity conditions specify when the switch configuration becomes inconsistent with the value of circuit variables.

The procedure for simulating the system is to calculate the state trajectory within each switch configuration, while numerically solving for the transition boundary to the next switch configuration. Because the state equations take on a simple form in this system, analytic expressions can be obtained for the state trajectories in each switch configuration. This eliminates the need for numerical integration, resulting in a computational advantage over other simulation methods which require it, such as that used in [9].

This approach is very similar to the one taken in [6], with a few basic differences. The equations of [6] are a reduced set of the state trajectory and validity equations used here. Because symmetry between the three phases is assumed, and only changes in bridge output current are tracked, the method used in [6] is simple and compact. However, the fact that it requires symmetry and only tracks one variable limits its usefulness. Because the piecewise model we use tracks all of the inputs, state variables, and boundary conditions, it can be used to investigate a variety of conditions, such as unbalanced voltages or reactances within a bridge, which cannot be studied with the reduced model in [6].

A computer program has been constructed to simulate the system in this manner, with full control over system parameters, firing angles, etc. In addition to constructing the detailed current waveforms in the system, the simulator uses a trapezoidal integration method to calculate the average magnetizing current during steady state operation. (Note that the integrator is only used to calculate the average current, and not the state trajectory.) This simulation method provides a more precise view of circuit operation than does the averaged model.

\section{RESULTS}

To make the results of the analysis most useful, we present them in terms of the following normalized parameters: $\bar{i}_{\mu} / I_{d}$ (relative current imbalance), $X_{c} I_{d} / V_{s}$ (commutating reactance factor), and $X_{\mu} I_{d} / V_{s}$ (magnetizing reactance factor). The state equations for the system can be directly normalized in this manner.

Piecewise model simulations verified the averaged model analysis in all cases where the approximation of small ripple was valid. Fig. 5 shows both simulation and analytic predictions of steady state current imbalance for different values of $\Delta \alpha$, while Fig. 6 shows the steady state current imbalance predicted by the two models when the two bridges have unequal ac source voltages. Fig. 7 compares the responses predicted by the two models for a step change in $\Delta \alpha$. As long as $\Delta \alpha$ varies at a rate slower than the ripple frequency, the simple first order model predicts the averaged current dynamics accurately.

An assumption used to develop the averaged model is that the magnetizing inductance is large enough that the effects 


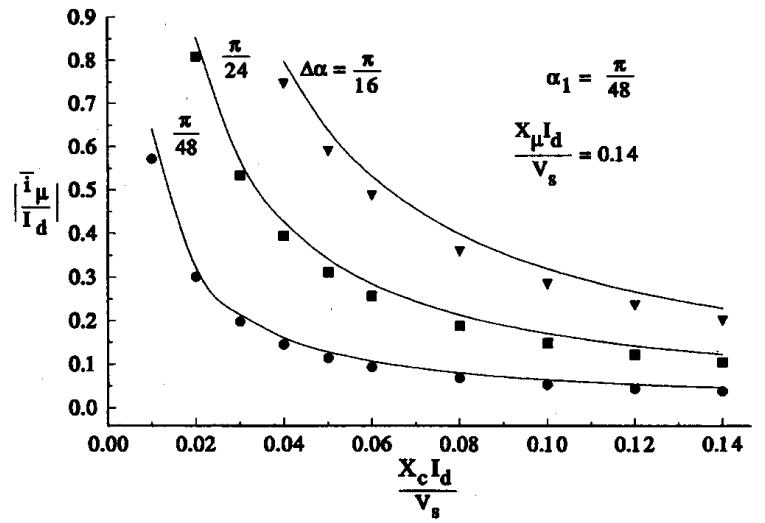

Fig. 5. Simulated and calculated current imbalance as a function of commutating reactance factor and $\Delta \alpha$. Data points are simulation results; solid lines are analytic results using the averaged model.

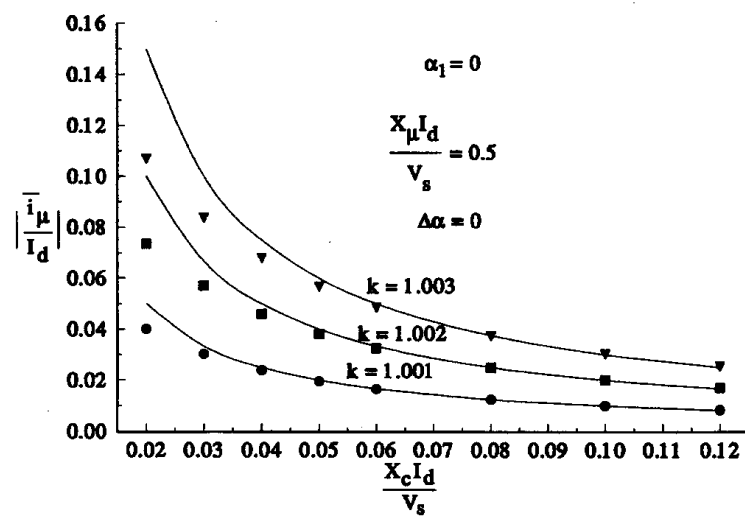

Fig. 6. Simulated and calculated current imbalance as a function of commutating reactance factor and $k$. Data points are simulation results; solid lines are the analytic results using the averaged model.

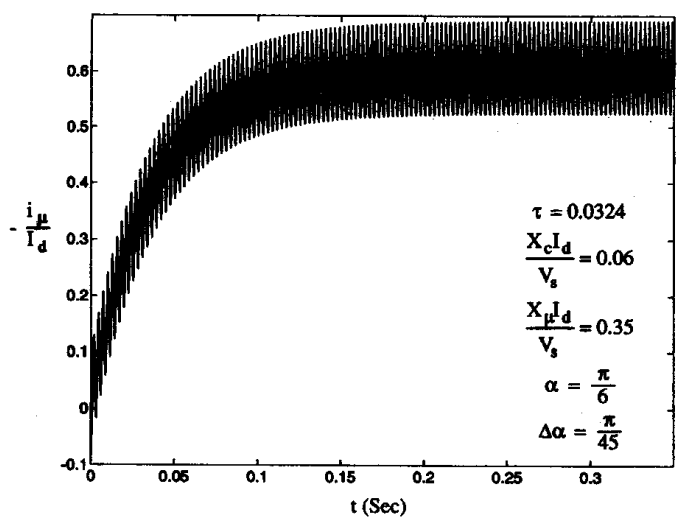

Fig. 7. Piecewise and averaged responses to a step in $\Delta \alpha$. The averaged response is the heavy line with no ripple.

of the current ripple in each bridge can be ignored. On the other hand, the piecewise simulations accurately predict the effects of nonzero current ripple. Fig. 8 illustrates the effect of this assumption on the accuracy of the averaged model results
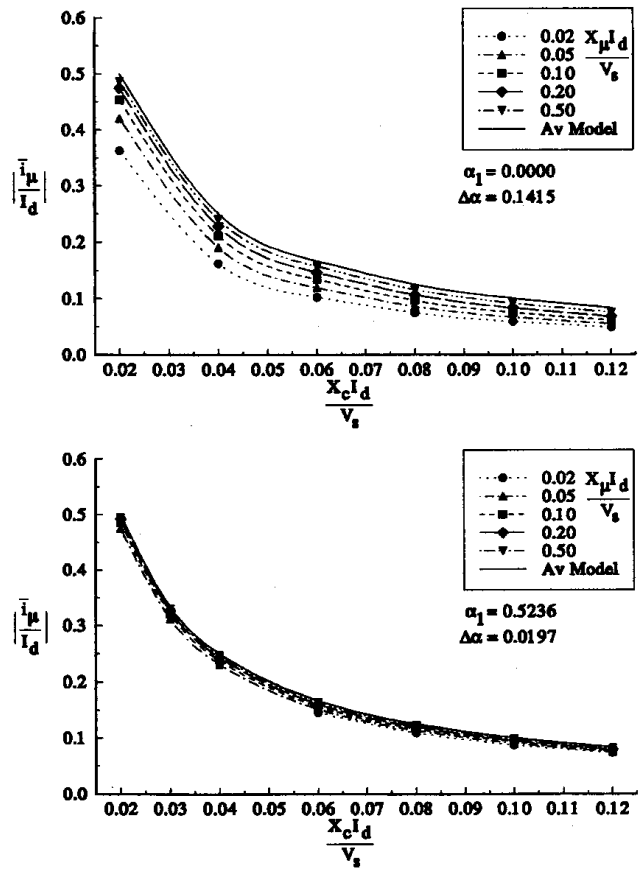

Fig. 8. Effects of varying the magnetizing reactance factor at two $\alpha_{1}$ 's.

by comparing them to those obtained by simulation over a range of magnetizing inductances. The data show that the averaged model accurately predicts the current imbalance as the magnetizing reactance factor becomes large. However, as this factor becomes small, significant deviations from averaged model predictions can result, especially at small values of $\alpha_{1}$. (As can be seen in (4), the sensitivity of the current imbalance to $\Delta \alpha$ is increased at small values of $\alpha_{1}$.) Thus, the conclusion of [6], that the size of the interphase reactance does not affect the average current imbalance, is not valid when $\alpha_{1}$ is small and the ripple current is large.

Errors in averaged model predictions of the average current imbalance are due to the model used for load regulation in a six-pulse bridge. This model assumes zero ripple in the bridge output current. But for finite magnetizing inductance, there is a nonzero ripple in $i_{\mu}$, and hence in the output current. This ripple affects the commutation times and, therefore, the load regulation of the bridges, resulting in the observed error. This effect is similar to the converter interactions analyzed by Freris for ac-side coupling [10], in that the coupling between converters significantly affects the load regulation phenomenon.

Fig. 9 illustrates current imbalance as a function of $\alpha_{1}$. An observation which can be drawn from this figure is that a given $\Delta \alpha$ will cause a much larger current imbalance as $\alpha_{1}$ is increased, with the most drastic increases occurring at small values of $\alpha_{1}$. What may be concluded from these observations is that for a system in which $\Delta \alpha$ can be no smaller than some minimum value, the current imbalance must be evaluated at the maximum $\alpha$ to be used (to $\alpha=90^{\circ}$ ). Furthermore, it is clear that in a system where $\alpha$ varies significantly from zero, the 


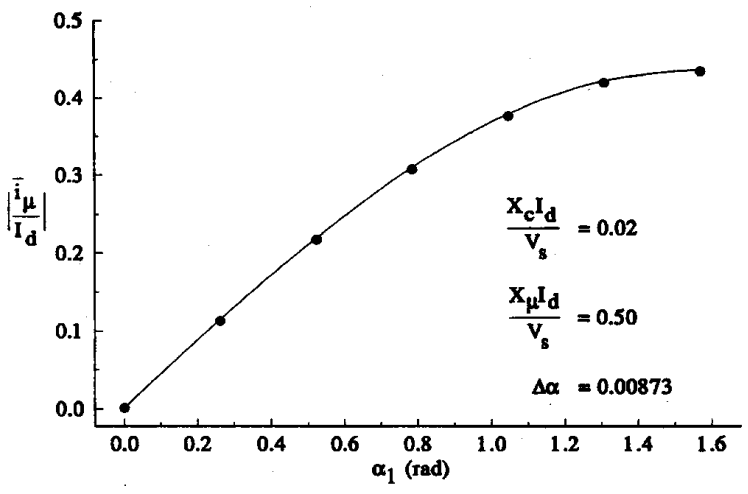

Fig. 9. Current imbalance as a function of $\alpha_{1}$.

feedback due to load regulation alone is of limited usefulness in controlling current imbalance.

The results presented here assume continuous conduction in each bridge. However, under conditions of large ripple and/or current imbalance, one of the bridge currents may become discontinuous. This is the reason some of the simulation curves are not extended to low values of $X_{c} I_{d} / V_{s}$. Analytic prediction of this effect is complicated, due to the interaction between bridges. Thus, when conditions of low magnetizing reactance factor and/or large current imbalances are encountered, piecewise linear modeling should be used to analyze the system. An effect which may occur under heavy load conditions is the transition of converter operation from mode 3 (single commutations in each bridge, nonoverlapping) to mode 4 (single commutations, overlapping) [9]. Mode 4 is entered when the commutation period of one of the bridges exceeds $\pi / 6$. In this case, average model predictions will still be valid, but piecewise modeling of the system becomes more complex.

\section{EXAMPLE}

It has been demonstrated that feedback control based on current imbalance, as shown in [6], or core flux, as shown in [11], can be effective for balancing currents in interphase transformers. The averaged model of Fig. 3 (or a variant including the load dynamics) is a useful tool for designing the feedback control, since it describes the averaged dynamics of the system. Under the constraint that the bandwidth of the control system is much lower than the switching frequency, control designs based on this model will yield correct results for low- frequency perturbations. The piecewise model can be used to verify the performance of the closed loop system.

We consider the design of a control loop for balancing the currents between bridges, as shown in the diagram of Fig. 10 . (With sufficiently slow load dynamics, control of load current can be treated separately on a longer time scale.) If we consider only differential-mode components in Fig. 3, we arrive at the differential-mode averaged circuit of Fig. 11. To drive the average magnetizing current to zero, the firing angle of the right hand bridge is delayed by an additional angle $\beta$, whose value is determined by the current-balance controller, yielding

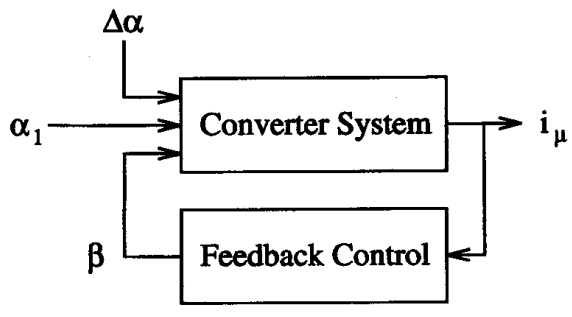

Fig. 10. Closed loop control block diagram.

$$
\begin{gathered}
2 \mathrm{R}_{\mathrm{eq}}=\frac{6 \omega \mathrm{L}_{\mathrm{c}}}{\pi} \\
\mathrm{v}_{\text {diff }}=\frac{3 \mathrm{~V}_{\mathrm{s}}}{\pi}\left[\cos \left(\alpha_{1}\right)-\cos \left(\alpha_{1}+\Delta \alpha+\beta\right)\right]
\end{gathered}
$$

Fig. 11. Differential-mode averaged circuit for current balance.

the dynamic relation (9).

$$
\begin{aligned}
\frac{d \bar{i}_{\mu}}{d t}= & -\frac{3 V_{s}}{\pi L_{\mu}} \sin \left(\alpha+\frac{\Delta \alpha}{2}+\frac{\beta}{2}\right) \\
& \cdot \sin \left(\frac{\Delta \alpha}{2}+\frac{\beta}{2}\right)-\frac{R_{e q}}{2 L_{\mu}} \bar{i}_{\mu} .
\end{aligned}
$$

Similar to (6), we consider PI control of the average magnetizing current. To eliminate the effects of current ripple and limit the bandwidth of the controller, a low-pass filtered version of $i_{\mu}$ is used for control, yielding

$$
\begin{aligned}
\beta & =\left(k_{p}+\frac{k_{i}}{s}\right) \frac{\omega_{c}}{s+\omega_{c}} i_{\mu} \\
& \approx\left(k_{p}+\frac{k_{i}}{s}\right) \frac{\omega_{c}}{s+\omega_{c}} \bar{i}_{\mu}
\end{aligned}
$$

where $\omega_{c}$ is set at one-tenth the ripple frequency.

This control law results in nonlinear closed-loop dynamics for the averaged model. However, for small perturbations in $\Delta \alpha$ and $\beta$, the control dynamics can be predicted using a linearized model. If we expand the nonlinear factors of (9) in Taylor series, and retain only linear terms, we find

$$
\frac{d \bar{i}_{\mu}}{d t} \approx-K \Delta \alpha-K \beta-\frac{R_{e q}}{2 L_{\mu}} \bar{i}_{\mu}
$$

where

$$
K=\frac{3 V_{s} \sin (\alpha)}{2 \pi L_{\mu}} .
$$

If the control law (10) is applied to the linearized system, we get the closed loop transfer function (13).

$$
\begin{gathered}
\frac{\bar{i}_{\mu}(s)}{\Delta \alpha(s)} \approx\left[-K s\left(s+\omega_{c}\right)\right] /\left[s^{3}+\left(\omega_{c}+\frac{R_{e q}}{2 L_{\mu}}\right) s^{2}\right. \\
\left.+\left(K k_{p} \omega_{c}+\frac{R_{e q} \omega_{c}}{2 L_{\mu}}\right) s+K k_{i} \omega_{c}\right]
\end{gathered}
$$




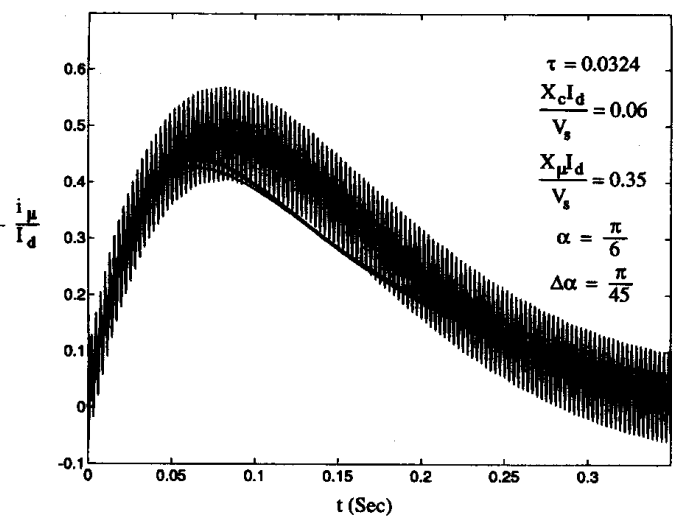

Fig. 12. Closed-loop response to a step in $\Delta \alpha$. The heavy line is the nonlinear model result; the light line is the linearized model result.

This transfer function is valid for low-frequency perturbations of sufficiently small size. For larger perturbations, the nonlinear model (9) and (10) should be used. For high frequency perturbations (approaching half the ripple frequency), the system becomes significantly time-varying because it only responds to changes in firing angle at specific times.

Consider a rectifier system with parameters $\omega=377 \mathrm{rad} / \mathrm{sec}$, $V_{s}=520 \mathrm{~V}, L_{c}=41.36 \mu \mathrm{H}$, and $L_{\mu}=241.24 \mu \mathrm{H}$, operating at $I_{d}=2000 \mathrm{~A}$, and $\alpha_{1}=\pi / 6$. This system has normalized parameters matching the example of Fig. 7 . We examine the closed-loop response to a $\pi / 45$ radian step in $\Delta \alpha$ with sample controller gains $k_{p}=1.5 \times 10^{-05}$, and $k_{i}=4.0$ $\times 10^{-04}$. Fig. 12 shows the responses for the piecewise-linear model, the nonlinear averaged model (9) and (10), and the linearized model (13). Both the linear and nonlinear averaged models do a good job of capturing the system dynamics, even though the perturbation waveform has significant highfrequency content. As can be seen from this example, the averaged models developed here can serve as a basis for current-balance compensator design.

\section{CONCLUSION}

The effects of firing angle imbalance between the sixpulse groups of a twelve-pulse rectifier have been examined. It has been shown that the amount of current imbalance is limited by the load regulation of the six-pulse groups. Equations have been developed to calculate the dynamics of the current balance using an averaged model. The effects of unbalanced turns ratios and commutating reactances have also been analyzed. These results have been verified via computer simulation using a piecewise model of the system.

\section{REFERENCES}

[1] F. T. Bennell, "Current balance in 12-pulse rectifiers comprising parallel bridges," in IEE Conf. Publ. 154, Power Electronics-Power Semiconductors and Their Applications, 1977, pp. 66-69.

[2] R. D. Evans, "Harmonics and load balance of multiphase rectifiers," AIEE Transactions, vol. 62, pp. 182-187, Apr. 1943.
[3] T. Subbarao and J. Reeve, "Harmonics caused by imbalanced transformer impedances and imperfect twelve-pulse operation in HVDC conversion," IEEE Trans. Power App. Sys., vol. 95, no. 5, pp. 1732-1735, Sept./Oct. 1976.

[4] R. Yacamini and J. C. de Olivera, "Core saturation transients caused by convertor firing unbalance," in Proc. IEE, vol. 126, no. 3, Mar. 1979, pp. 233-238.

[5] H. von Bertele and H. Grasl, "Anomalies in converter transformer operation," Direct Current, pp. 203-214, Aug. 1962.

[6] T. Tanaka, S. Ogasawara, Y. Akagi, and A. Nabae, "Current balance of phase-controlled thyristor converter with interphase reactors," Electrical Eng. Japan, vol. 110, no. 4, pp. 83-91, 1990.

[7] J. G. Kassakian, M. F. Schlecht, and G. C. Verghese, Principles of Power Electronics. Reading, MA: Addison-Wesley, 1991, pp. 261-274.

[8] —, Principles of Power Electronics. Reading, MA: AddisonWesley, 1991, p. 45 .

[9] J. K. Hall, J. G. Kettleborough, and A. B. M. J. Razak, "Parallel operation of bridge rectifiers without an interbridge reactor," in Proc. IEE Pt. B, vol. 137, no. 2, Mar. 1990, pp. 125-140.

[10] L. L. Freris, "Effects of interaction among groups in a multigroup a.c.-d.c. convertor," in Proc. IEE, vol. 114, no. 7, July 1967, pp. 965-973.

[11] F. P. Dawson, "DC-DC converter interphase transformer design considerations: Volt-seconds balancing," IEEE Trans. Magnetics, vol. 126, no. 5, pp. 2250-2252, Sept. 1990.

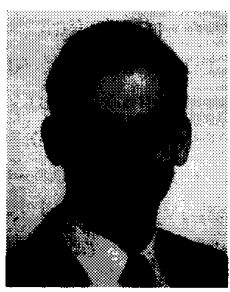

David J. Perreault (S'91) was born in North Providence, RI, on January 22, 1967. He received the B.S. degree from Boston University, Boston, MA, in 1989, and the M.S. degree from the Massachusetts Institute of Technology, Cambridge, in 1991, both in electrical engineering.

$\mathrm{He}$ is currently a Ph.D. candidate at MIT and a Research Assistant in the MIT Laboratory for Electromagnetic and Electronic Systems, where he is engaged in research and development of cellular power electronic architectures.

Mr. Perreault is a member of Tau Beta Pi and the National Society of Professional Engineers, and was the recipient of the IEEE Convergence Fellowship in Transportation Electronics.

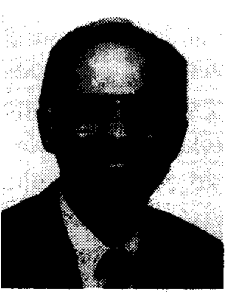

John Kassakian (S'65-M'73-SM'80-F'89) is Professor of Electrical Engineering and Director of the MTT Laboratory for Electromagnetic and Electronic Systems.

He has been teaching and doing research in power electronics since 1973, and serves on the Board of Directors of several companies in the electronics industry. He has published extensively in the area of power electronics, and is a co-author of the textbook Principles of Power Electronics.

Dr. Kassakian was the founding President of the IEEE Power Electronics Society, serves as the United States representative to the European Power Electronics Society, and was the recipient of the IEEE Centennial Medal, the IEEE William E. Newell Award, and the Distinguished Service Award from the IEEE Power Electronics Society. In 1989, he was elected a Fellow of the IEEE and in 1993, he was elected to the National Academy of Engineering, and was also awarded an IEEE Distinguished Lectureship, through which he has lectured internationally. 